\title{
An international, multicenter, observational survey to evaluate diabetes control in subjects using insulin for the treatment of type I and type 2 diabetes mellitus in the Czech Republic and Slovak Republic: study protocol for a cross-sectional survey
}

This article was published in the following Dove Press journal:

Open Access Journal of Clinical Trials

9 June 2016

Number of times this article has been viewed

\author{
Jan Brož' \\ Denisa Janickova Zdarska' \\ Jana Urbanova ${ }^{2}$ \\ Marek Brabec ${ }^{3}$ \\ Bohumila Krivska ${ }^{4}$ \\ Viera Donicova ${ }^{5}$ \\ Radka Stepanova ${ }^{6}$ \\ Emil Martinka ${ }^{7}$ \\ Milan Kvapil'
}

'Department of Internal Medicine, Second Faculty of Medicine, ${ }^{2}$ Center for Research on Diabetes, Metabolism and Nutrition, Second Department of Internal Medicine, Third Faculty of Medicine, Charles University, ${ }^{3}$ Institute of Computer Science of the ASCR, vvi, ${ }^{4}$ Sanofi, Prague, Czech Republic; ${ }^{5}$ Private Department of Diabetology, Internal Medicine and Metabolism, Kosice, Slovak Republic; ${ }^{6}$ ADDS sro, Brno, Czech Republic; ${ }^{7} \mathrm{National}$ Institute of Endocrinology and Diabetology, Lubochna, Slovak Republic
Correspondence: Jan Brož

Department of Internal Medicine, Second Faculty of Medicine, Charles University, V Úvalu 84, 15006 Prague, Czech Republic

Email zorb@seznam.cz
Background: Despite the improvements in insulin therapy, a large number of patients fail to achieve their target glycated hemoglobin (HbAlc) levels. Control of diabetes is often unsatisfactory because the patient does not know about the principles of successful insulin therapy (ie, blood glucose self-monitoring, the principles of insulin administration, titration, current dose adjustments, dietary recommendations, and physical activity preventive measures) or because these principles are applied incorrectly or insufficiently. Furthermore, the fear of hypoglycemia may lead to maintaining higher than recommended blood glucose levels.

Methods/design: This is a noninterventional, international study focusing on a questionnaire survey of diabetes patients (patient-reported outcome) treated with insulin for at least 1 year. It is designed so that the data obtained reflect real access of patients to insulin treatment. The primary objective is to show the results of glycemic control of diabetes (HbAlc) achieved in diabetes patients treated with at least one dose of insulin. The secondary objective is to monitor the factors potentially affecting these results, which include the frequency and other characteristics of hypoglycemia, the frequency of blood glucose self-monitoring, and the effects produced when the results are employed in adjusting the therapy. Furthermore, the study investigates factors related to the principles of insulin administration, dietary regime, and exercise habits. The study will enroll a total of 1,500 patients with type 1 and type 2 diabetes in 150 centers: two-thirds in the Czech Republic and one-third in the Slovak Republic.

Discussion: The study is primarily aimed at determining the percentage of insulin-treated diabetes patients reaching the recommended targets for glycemic control (HbAlc). Furthermore, it attempts to identify and describe in detail the factors of failure in achieving the therapeutic goals. An analysis of the data thus obtained may result in recommendations on how to reduce and eliminate all the identified negative factors in the future.

Keywords: insulin therapy, glycemic control, HbA1c, hypoglycemia, education, diabetes regimen adherence

\section{Background}

The DCCT and UK Prospective Diabetes Study ${ }^{1,2}$ trials have shown that achieving the recommended blood glucose targets reduces morbidity in patients with diabetes mellitus (DM). Standards of medical care for diabetic patients defined by the American Diabetes Association (ADA) recommend to reduce the glycated hemoglobin (HbA1c) 
levels $<7.0 \%$ ( $<53 \mathrm{mmol} / \mathrm{mol})$. This leads to a decline in the incidence of microvascular complications in the majority of patients. ${ }^{3}$ More stringent treatment goals $(<6.5 \%)$ can be proposed, namely, in patients with a short duration of diabetes, long life expectancy, and no cardiovascular disease, if these can be achieved without hypoglycemia or other adverse effects of treatment. Conversely, higher than the recommended $\mathrm{HbA} 1 \mathrm{c}$ levels $(7.5 \%-8.0 \%)$ can be tolerated on an individual basis in the following three groups of patients: first, in patients with a history of severe hypoglycemia and advanced micro- and macrovascular complications; second, in those with a short life expectancy; and third, wherever maximum care, including extensive diabetes education, adequate blood glucose self-monitoring, and the use of combined treatment with available drugs, including insulin, proves inefficient. These recommendations are supported by the consensus opinion of the European Association for the Study of Diabetes and the ADA. ${ }^{4,5}$

Nevertheless, the DEPAC trial, conducted at outpatient diabetes clinics in eight countries of Central and Eastern Europe (the Czech Republic, Estonia, Hungary, Lithuania, Latvia, Poland, Slovak Republic, and Slovenia), showed that only a minority of DM patients (13.1\% for type $1 \mathrm{DM}$; $21.4 \%$ for type $2 \mathrm{DM}$ ) achieved their defined therapeutic goals (HbA1c <6.5\%), with mean HbA1c levels of $8.2 \%$ for type $1 \mathrm{DM}$ and $7.7 \%$ for type $2 \mathrm{DM}^{6}$

Adequate glycemic control is the main priority of DM treatment strategy. HbAlc level $\geq 7.0 \%$ indicates necessary therapeutic changes so as to improve the treatment outcomes, ie, to achieve a decrease in HbAlc values. If patients fail to achieve the target $\mathrm{HbA} 1 \mathrm{c}$ levels, they are advised to come for repeated medical consultations and their therapy is changed toward a combination of antidiabetic drugs, including insulin, taking into account the indication differences in the treatment of various types of diabetes. Stronger emphasis is laid on diabetes education in order to substantially improve the patients' dietary habits, blood glucose self-monitoring, knowledge about hypoglycemia, and exercise habits.

Medical care for patients with diabetes includes both the administration of medicines and a range of nonpharmacological modalities subject to careful and repeated education. Lifestyle changes should include dietary adjustments; an increase in regular, adequate exercise; and finally, acquiring insulin therapy-related skills in patients already treated with insulin. Insulin therapy is an essential part of treatment in type 1 DM patients' therapy and an option in patients with type 2 DM. For insulin therapy to provide the desired $\mathrm{HbA} 1 \mathrm{c}$ results, the patients are required to have specific knowledge and proficiency in a number of therapeutic tasks. Their sufficient integration into the patients' everyday life is subject to regular checkups and repeated diabetes education. $^{7}$

Several factors related to the disease itself or associated with the patient and/or physician are known to contribute to poor glycemic control. In the case of the illness itself, it may be affected by hypoglycemia-related autonomic failure consisting of an inadequate bodily response to hypoglycemia caused by defective contraregulatory mechanisms that are associated with inadequate adrenaline and glucagon response to incipient hypoglycemia. The patient fails to recognize the condition that leads to the risk of higher frequency of hypoglycemia, including severe hypoglycemia. The patient then preventively maintains higher than the recommended blood glucose levels. Sometimes, this behavior aimed at strictly minimizing the risk of hypoglycemia occurs on the basis of unpleasant sensations induced by hypoglycemia, in particular if this event was difficult to cope with. ${ }^{8}$ Fears of hypoglycemia, in the earlier context, may also be associated with poor knowledge of the prevention and treatment. ${ }^{9}$ Insufficient knowledge about blood glucose self-monitoring, dietary restrictions, insulin dosage regimen, insulin titration, and actual adjustments of the doses as much as about preventive tasks related to physical activity ${ }^{10-12}$ may also affect the results of treatment in a negative way. However, the extent to which the earlier factors contribute to poor DM control, namely, those directly associated with insulin therapy, has not yet been closely examined in full context.

The effects of diabetes on the quality of life in patients treated with insulin in the Czech and Slovak Republics have not yet been studied in detail. A former study suggested that the quality of life influenced by diabetes (the Audit of Diabetes-Dependent Quality of Life [ADDQoL19]) is negatively correlated with $\mathrm{HbA} 1 \mathrm{c}{ }^{13}$

\section{Aim}

The study aims to evaluate glycemic control as measured by $\mathrm{HbA} 1 \mathrm{c}$ in patients with type 1 and type $2 \mathrm{DM}$ treated with at least one insulin injection daily, and, at the same time, to describe and put into context factors affecting the achievement of this control.

These factors include the frequency and other characteristics of hypoglycemia, the frequency of blood glucose self-monitoring, and the use of the results in treatment together with the factors related to the principles of insulin administration, diet regimen, exercise habits, and efficient education. 
Hypoglycemia may be associated with other adverse clinical and economic factors that can produce a negative effect on the results of treatment. Therefore, the study also defines a number of secondary end points, referring specifically to this phenomenon. These are in particular the frequency of hypoglycemia episodes, their causes, and the methods and success in their management by the patient together with their influence on various aspects of the patient's life. Furthermore, the secondary end points include monitoring the dietary habits of the patients, their titration skills, and capability to adjust the current dose of insulin together with the adherence to the schedule of insulin administration. Moreover, it is investigated how often they take blood glucose measurements using a self-monitoring device and, last but not the least, what is their approach with respect to guidelines on therapeutic exercise. The study also investigates the level of diabetes education provided to the patients.

The secondary objectives comprise monitoring cardiovascular risk factors and detecting the latest values of basic laboratory parameters in this area.

The study evaluates the rate with which diabetes influences the quality of life in these patients and determines its possible correlation with the achieved glycemic control.

\section{Methods/design}

\section{Research design}

This is an international, observational study that focuses on a questionnaire survey of diabetes patients (patient-reported outcome) treated with insulin. It contains a retrospective and a prospective phase. The study design will reflect the patients' actual approach to the treatment of diabetes with insulin. A total of 1,500 patients will be enrolled at 150 study centers (100 in the Czech Republic, 50 in the Slovak Republic). Centers will be selected based on outpatient diabetologists who have experience with insulin treatment, respecting the distribution in all the regions of the Czech Republic and Slovak Republic to achieve the highest possible representativeness.

\section{Outcomes}

The primary objective is to determine the prevalence of diabetes patients on insulin treatment and achieving $\mathrm{HbA} 1 \mathrm{c}$ $<7.0 \%$ (DCCT), obtained in the enrollment visit (V1).

The secondary objectives are to determine the incidence of hypoglycemia as per the ADA definitions, ${ }^{14}$ determine their causes and methods that the patients use to manage them; determine the frequency of insulin titration, insulin dose adjustments, and adherence to the time schedule of administration on the part of the patients; determine the frequency of blood glucose self-monitoring; and determine the level of education in other areas, especially with regard to diet and exercise habits. All the secondary objectives set are listed in Table 1.

\section{Sample size calculation}

A total of 1,000 patients in the Czech Republic and 500 patients in the Slovak Republic are planned to be enrolled in the study. No discontinuation of patients is expected due to failure to complete the study, and all the patients enrolled will be used for analysis to determine the proportion of diabetes patients with $\mathrm{HbA} 1 \mathrm{c}<7.0 \%$ at the $\mathrm{V} 1$. The proportion of patients who will completely fill the study questionnaires and patients diary is not predictable, and it is one of the objectives of this study.

The proportion of insulin-treated patients with type 1 and type 2 diabetes is expected to be 1:3, which corresponds to 250:750 evaluable patients in the Czech Republic and 125:375 evaluable patients in the Slovak Republic. The percentage of patients with type 2 diabetes treated with premixed insulin, intensified insulin regimen, and basal insulin + noninsulin products is expected to be $\sim 30 \%$ in each of these groups. The percentage of other types of insulin treatment is expected to be $<10 \%$.

Analysis of the number of patients in each group will allow determination of the frequency of patients with $\mathrm{HbA} 1 \mathrm{c}$ levels in the target range with sufficient accuracy (two-sided 95\% Wilson score CI) for this type of study, with $10.6 \%$ accuracy for type 1 and $6.4 \%$ accuracy for type 2 diabetes in the Czech Republic and 14.8\% accuracy for type 1 and $9.2 \%$ accuracy for type 2 diabetes in the Slovak Republic. Since

Table I Secondary objectives

Incidence of hypoglycemia according to ADA definitions Methods of coping with hypoglycemia

Frequency of blood glucose self-monitoring Causes of hypoglycemia and their frequency Symptoms of hypoglycemia and their frequency Changes in insulin dosage in relation to hypoglycemia and their frequency

Frequency of insulin titration and changes in insulin dosage Level of diabetes education and frequency of counseling Assessment of basic cardiovascular risk factors

Weight changes in the course of diabetes and the current weight Development of treatment and contemporary treatment of diabetes Development of $\mathrm{HbAlc}$ in the course of diabetes Quality of life based on the ADDQoL questionnaire

Abbreviations: ADA, American Diabetes Association; HbAlc, glycated hemoglobin; ADDQoL, Audit of Diabetes-Dependent Quality of Life. 
no relevant published data have been available, qualified estimates of the number of patients who will achieve the recommended target values of $\mathrm{HbA} 1 \mathrm{c}$ were used to calculate the sample size (expected percentage), namely, 20\% of patients with type 1 diabetes and $25 \%$ of patients with type 2 diabetes.

\section{Inclusion and exclusion criteria}

The inclusion criteria are type 1 or type 2 diabetes, patient aged $\geq 18$ years, treatment with at least one insulin injection daily for a period $>1$ year, willingness to fill in the study questionnaires, and a signed patient's informed consent form.

The exclusion criteria are insulin therapy for a duration of $<12$ months, current insulin pump therapy or use of this treatment in the last 12 months, unavailability of the test result of $\mathrm{Hb} 1 \mathrm{Ac}$ in the last 3 months before the V1, pregnancy, inability to be present at the follow-up visit (V2), inability to complete the study questionnaires, and participation in another clinical study within the last 3 months.

\section{Eligibility assessment and $\mathrm{V} 2 \mathrm{~s}$}

Eligibility and exclusion criteria will take place during the V1 and will be assessed by medical records, patient interviews, and laboratory tests.

\section{Study sample}

Doctors will offer participation in the study to all consecutive type 1 and type 2 diabetic patients treated with insulin for at least 1 year until the quota for the respective center has been filled. The aim is to recruit ten patients in each center with a maximum of 14 patients per center in case of inadequate recruitment in some centers. The total number of centers will be 150 (100 in the Czech Republic and 50 in the Slovak Republic).

\section{Data collection}

Data will be recorded during two routine visits of patients (V1 and V2).

\section{Enrollment visit}

During the V1, the physician will evaluate the inclusion and exclusion criteria. The physician will ask the patient whether he/she is willing to participate in the project and will make the patient sign the informed consent form. Patients included in the study will receive a set of questionnaires from the doctor (diabetes control in subjects using insulin for the
Table 2 Data recorded by the doctor during the enrollment visit

Inclusion/exclusion criteria

Age

Sex

Height

Body weight

BMI

Smoking status

SBP, DBP, and heart rate

Personal medical history

Current and past histories of diseases

Pharmacological history

Concomitantly used medicines

Date of diagnosis of DM

Information on the concomitant and previous treatment with noninsulin antidiabetic drugs, if any

Information on the initiation of insulin treatment

Complications of diabetes

HbAlc levels

Fasting blood glucose

Lipid profile (total cholesterol, HDL, LDL, and TG)

Uric acid, creatinine, TSH, anti-TPO, and microalbuminuria (results of

examination, if performed within the normal treatment process)

Body weight measurements obtained in the last 3 years

$\mathrm{HbAlc}$ levels obtained in the last 3 years

History of insulin and noninsulin treatment of diabetes obtained in the last 3 years

Abbreviations: BMI, body mass index; SBP, systolic blood pressure; DBP, diastolic blood pressure; DM, diabetes mellitus; HbAIc, glycated hemoglobin; HDL, highdensity lipoprotein; LDL, low-density lipoprotein; TG, triglycerides; TSH, thyroidstimulating hormone; TPO, thyroid peroxidase antibodies.

treatment [DIAINFORM], ADDQoL19, Diabetic Diary). The DIAINFORM and ADDQoL19 questionnaires will be filled in by the patients during the V1, while the Diabetic Diary will be completed between the V1 and V2, optimally within 4 weeks following the V1. The data reported by the doctor will be obtained from the medical records and will be recorded in the electronic case report forms.

The data to be obtained during the V1 are specified in Tables 2 and 3 .

\section{Follow-up visit}

A V2 will take place within the usual clinical practice after $\sim 3$ months of the V1. During this visit, the patient will submit his/her completed Diabetic Diary. The data to be obtained during the V2 are specified in Table 4.

Table 3 Patient-reported outcomes at the enrollment visit

Quality of life questionnaire ADDQoL

Retrospective questionnaire DIAINFORM

Abbreviations: ADDQoL, Audit of Diabetes-Dependent Quality of Life; DIAINFORM, diabetes control in subjects using insulin for the treatment. 
Table 4 Data recorded by the physician during the follow-up visit

Body weight

BMI

SBP, DBP, and heart rate

Potential changes in the diabetes and concomitant medication

$\mathrm{HbAlc}$

Fasting glycemia

Record on completion of the Diabetic Diary

Abbreviations: BMI, body mass index; SBP, systolic blood pressure; DBP, diastolic blood pressure; HbAlc, glycated hemoglobin.

\section{Key instruments (questionnaires)}

The following questionnaires are used in the study.

\section{DIAINFORM questionnaire}

The DIAINFORM questionnaire was developed by the study research team to obtain data describing significant areas associated with insulin therapy and diabetes in general. These areas include hypoglycemia, insulin titration, the current dose adjustments of insulin, compliance with the insulin administration regimen, blood glucose selfmonitoring, and dietary and exercise habits. The largest part of the questionnaire is devoted to hypoglycemia in order to determine its frequency, severity, symptoms, patients' recognition of hypoglycemia, treatment methods and success, possibilities of prevention, and their impact on the patient's quality of life. The questionnaire is included in the protocol (Supplementary material).

\section{ADDQoLI9 questionnaire}

It is a standard and proven tool for validation of the impact of diabetes on the quality of life of patients. ${ }^{13}$ The official Czech and Slovak language versions are used in the study.

\section{Diabetic Diary}

The Diabetic Diary was developed by the study research team as a tool for prospective monitoring of the frequency of hypoglycemic episodes and some parameters associated with them. It is a diary in which the patient will record, for the period of 1 month, the circumstances associated with hypoglycemic episodes, which are mainly the symptoms, the time of hypoglycemia in relation to wakefulness or sleep or to the work process (job), the causes of hypoglycemia, and the success of their treatment. Information on nonadherence to the time schedule of the insulin administration and any abnormalities in the diet and physical activity will also be recorded in the Diary.

\section{Study interventions}

The study is noninterventional.

\section{Statistical analysis plan}

\section{Primary analysis}

The primary objective of the study is to determine the percentage (prevalence) of insulin-treated diabetic patients with HbA1c levels $<7.0 \%$ (for patients with diabetes type 1 and type 2 separately). The prevalence will be calculated as a percentage of the patients with $\mathrm{HbA} 1 \mathrm{c}$ levels $<7.0 \%$ (obtained in the V1) accompanied by two-sided $95 \%$ CI.

\section{Secondary analysis}

In the secondary analysis, the prevalence of patients with $\mathrm{HbA} 1 \mathrm{c}<7.0 \%$ will be assessed in the following treatment regimen groups for patients with type 2 diabetes: premixed insulin, intensified insulin treatment, basal insulin and noninsulin products, and other regimen.

Secondary analyses will also focus on the evaluation of hypoglycemia, blood glucose self-monitoring, insulin treatment, education about diabetes, changes in $\mathrm{HbAlc}$, weight change, quality of life, and emotional state. Analyses will be performed both for the entire population and independently for the specified groups of treatment regimens.

Data obtained from the questionnaires, patient diaries, and medical records will be presented descriptively in the form of frequency tables. Summary statistics will be used to evaluate continuous data. Changes in the examined continuous parameters will be tested using the Wilcoxon test or paired $t$-test. The paired McNemar's test will be used for binary data.

\section{Discussion Glycemic control (HbAlc)}

The latest study evaluating the level of glycemic control achieved by diabetes patients in the Czech Republic and Slovak Republic was carried out as part of a comparison between the eight new European Union countries in 2007. Specific data for the respective countries have not been published yet. In all eight countries, the then-recommended goal (HbA1c $<6.5 \%$, DCCT) was achieved on average only by $13.1 \%$ of patients with type 1 diabetes and $21.4 \%$ of patients with type 2 diabetes. The mean HbAlc level achieved was $8.2 \%$ for type 1 diabetes and $7.7 \%$ for type 2 diabetes. According to DCCT, the best and worst mean $\mathrm{HbA} 1 \mathrm{c}$ levels achieved in the said countries were $7.7 \%$ and 
$9.8 \%$, respectively, for patients with type 1 diabetes and $7.5 \%$ and $8.3 \%$, respectively, for patients with type 2 diabetes. Glycemic control in patients with type 2 diabetes treated with insulin or oral combination therapy with insulin has not been explored in detail in these countries. ${ }^{6}$

Glycemic control (not only in insulin-treated but also in other patients) is influenced by many factors, which for the sake of simplification we can call "external" (dependent on external circumstances, eg, the development of new drugs, their changing availability, changes in the organization and availability of care, etc) and "internal" (depending more on patients). External factors that could affect the achievement of glycemic control and that have been changed in the Czech Republic since 2007 are the following: increase in the number of patients with diabetes (from 2007 to 2012, the number of registered patients increased from 754,961 to 841,227 ), increase in the number of physicians' jobs in the management of diabetes (from 323.37 to $\sim 360.83$ at the corresponding ratio), and a slight increase in the number of nursing jobs (from 368.11 to 396.51). At the same time, there was an increase in the number of patients treated with insulin and insulin combined with oral antidiabetic treatments (from 188,202 in 2007 to 202,926 in 2012). Simultaneously, the number of patients treated with metformin (alone and in combination) also increased significantly over the same time period, and new classes of antidiabetic drugs were discovered, which may be used as a part of combination treatment with oral antidiabetic drugs and insulin in the treatment of type 2 diabetes. ${ }^{15}$ It would be rather speculative to evaluate the effects of these changes on glycemic control, but we can assume that more aggressive treatment (increased insulin therapy) and wider opportunities in the combination of oral antidiabetic agents with insulin outweigh the potentially negative effects of the overall increase in the number of patients and the number of patients treated with insulin, as well as the relative stagnation in the number of diabetesspecialized health care workers, especially in the number of nurses who often play the role of educators in the outpatient practice.

Another important external factor that could indirectly affect the final glycemic control is the greater availability of test strips for blood glucose measurements. Since the price of the test strips decreased to almost one half compared to that in the year 2007 and there were also changes in the reimbursement of the strips from the public health insurance schemes (to a certain extent, the strips are also reimbursed to patients with less than three daily administrations of insulin), an increase in the frequency of blood glucose self-monitoring can be expected, which can have a positive effect on glycemic control in the groups of patients being monitored. ${ }^{16-18}$

In addition to physiological and pathophysiological factors (higher body mass index, longer duration of diabetes, younger age, which are associated with higher $\mathrm{HbA} 1 \mathrm{c}$ levels in patients with type 2 diabetes), ${ }^{19}$ the internal factors influencing the glycemic control also include a group of factors that could be called skills and are related mainly to insulin therapy. These factors include, in particular, adherence to dietary recommendations and restrictions, conditions associated with hypoglycemic episodes (see later), the frequency and use of self-monitoring, adherence to insulin therapy, and sufficient physical activity. ${ }^{10-12,19}$ The objective of the study in this respect is to confirm the influence of the earlier factors and to try to determine more accurately their contribution to the achievement of glycemic control.

The contribution of preprandial insulin analogs to glycemic control compared to human insulin is unclear. ${ }^{20,21}$ Similarly unclear is the contribution of routinely used basal analogs, and the results of the studies cited herein, perhaps, slightly favor insulin glargine, especially with respect to type 2 diabetes. ${ }^{22-24}$

This observational study could help answer these questions as well as questions related to the results of glycemic control for different types of insulin regimens in combination with oral antidiabetic agents in the treatment of type 2 diabetes.

The intention to correlate some of the data obtained from the questionnaires with the $\mathrm{HbAlc}$ value brought about exclusion of patients with the HbAlc value determined earlier than 3 months prior to V1. Since it is common practice to determine the $\mathrm{HbA} 1 \mathrm{c}$ value approximately within 1 week preceding a medical checkup and the recommended frequency of determining the $\mathrm{HbA} 1 \mathrm{c}$ value in patients treated with insulin or insulin and peroral antidiabetic drugs is at least four times a year, we do not assume that this exclusion criterion may impact the evaluation of the real prevalence of the primary end point.

\section{Hypoglycemia}

Hypoglycemia is an adverse effect of some antidiabetic treatments (especially, insulin, sulphonylureas, and glinides), which may lead to personal injury, brain damage, and even death. In line with previous studies, we expect a higher frequency of hypoglycemic episodes in patients with type 1 diabetes compared to type 2 diabetes, including severe hypoglycemia, even when using a treatment with the same insulin regimen. We expect an increasing frequency of hypoglycemia 
with age and duration of diabetes within each of the subgroups defined by the type of diabetes and type of treatment. ${ }^{25,26}$

The study has been designed to determine the frequency of hypoglycemia in routine clinical practice with regard to different types of insulin and various types of insulin regimens and their combinations with oral antidiabetic agents. It should also demonstrate the correlation between the frequency of hypoglycemia and HbA1c levels, the frequency of severe hypoglycemia, and how hypoglycemia affects the patient's adherence to the treatment and his/her quality of life. In addition, the study also monitors the level of awareness of the warning symptoms of hypoglycemia. We will also record the frequency of hypoglycemia resulting in acute medical intervention, hospitalization, and/or injury and the frequency of hypoglycemia while driving a motor vehicle.

\section{Cardiovascular risk factors}

The study follows up the basic cardiovascular risk factors, such as blood lipid values, blood pressure, body mass index, smoking, physical activity and exercise, and the current and past history of cardiovascular diseases. In 2007 in the eight new EU countries (including the Czech Republic and the Slovak Republic), the target blood pressure values and low-density lipoprotein-cholesterol levels were achieved on average by $20 \%$ of patients with type 2 diabetes and by $50 \%-60 \%$ and $36 \%$, respectively, of patients with type 1 diabetes (data for individual countries were not published). ${ }^{6}$ Owing to cheaper prices and the elimination of prescribing restrictions for certain drugs and because of new drugs that can be now used, in particular for the treatment of blood pressure, we assume the target lipid and blood pressure values will be achieved in a higher percentage of patients.

\section{Quality of life}

Quality of life has never been studied among insulin-treated patients in the Czech Republic or the Slovak Republic. From recent studies in countries similar to the Czech Republic or the Slovak Republic, only the results of a study conducted in Slovenia are available, where only $3.9 \%$ of respondents $>66$ years of age with type 2 diabetes reported no impact of the disease on their quality of life. ${ }^{27}$ Similar results can be expected in our study. The study will also attempt to verify the recently published result that the diabetes-related quality of life is independently associated with the HbA1c level. ${ }^{27}$

\section{Ethics and data protection issues}

Participation will be voluntary and will follow the ethical aspects of confidentiality and data protection. The procedures will be explained to the patients, and information about the aim, design, associated potential risks and benefits, and all relevant details of the research will be given in the informed consent form. All patients will have to sign the informed consent form prior to participating in the study. The data obtained by the study will be available to the participant and to any other authorized person, and may be used anonymously for academic and scientific purposes. The study protocol was approved by the Ethics Committee of National Institute of Endocrinology and Diabetology in Lubochna, Slovak Republic (approval date February 4, 2015).

\section{Acknowledgments}

This study is funded by Sanofi-Aventis sro. The role of the sponsor was also to appoint an independent research company, which will carry out the study (ADDS sro).

\section{Disclosure}

JB has received payment for being an adviser to and a speaker for Sanofi-Aventis sro and Eli Lilly sro. He also received payment for being the international coordinator of the study. DJZ disclosed receipt of the financial support for the research, authorship, being a speaker, and being an educator from Sanofi-Aventis, AstraZeneca, Eli Lilly, Novo Nordisk, Merc Sharp and Dohme, and Johnson \& Johnson. BK is an employee of Sanofi, the Czech Republic. RS works as a statistician for CRO ADDS sro that is contracted by SanofiAventis sro for statistical analysis of this study. The authors report no other conflicts of interest in this work.

\section{References}

1. DCCT Research Group. The effect of intensive treatment of diabetes on the development and progression of long-term complications in insulin-dependent diabetes mellitus. $N$ Engl J Med. 1993;329: 977-986.

2. Stratton IM, Adler AI, Neil HA, et al. Association of glycaemia with macrovascular and microvascular complications of type 2 diabetes UK prospective diabetes study (UKPDS 35): prospective observational study. Br Med J. 2000;321(7258):405-412.

3. ADA. Standards of medical care in diabetes-2011. Diabetes Care. 2011;34(Suppl 1):S11-S61.

4. Inzucchi SE, Bergenstal RM, Buse JB, et al. Management of hyperglycaemia in type 2 diabetes: a patient-centered approach. Position statement of the American Diabetes Association (ADA) and the European Association for the Study of Diabetes (EASD). Diabetologia. 2012;55(6):1577-1596.

5. American Diabetes Association. Standards of medical care in diabetes 2014. Diabetes Care. 2014;37(Suppl 1):514-580.

6. Andel M, Grzeszczak W, Michalek J, et al; DEPAC Group. A multinational, multi-centre, observational, cross-sectional survey assessing diabetes secondary care in Central and Eastern Europe (DEPAC Survey). Diabet Med. 2008;25(10):1195-1203.

7. Cryer PE. Hypoglycemia-associated autonomic failure in diabetes. Am J Physiol Endocrinol Metab. 2001;28:1115-1121. 
8. Anarte MT, Carreira M, Machado A, et al. Identification of risk factors for suffering fear of hypoglycemia in type 1 diabetes mellitus patients. Scand J Psychol. 2014;55(6):554-557.

9. Frier BM. Hypoglycaemia in diabetes mellitus: epidemiology and clinical implications. Nat Rev Endocrinol. 2014;10(12):711-722.

10. Mashitani T, Hayashino Y, Okamura S, et al. Patient-reported adherence to insulin regimen is associated with glycemic control among Japanese patients with type 2 diabetes: Diabetes Distress and Care Registry at Tenri (DDCRT 3). Diabetes Res Clin Pract. 2013;100(2):189-194.

11. Brod M, Rana A, Barnett AH. Adherence patterns in patients with type 2 diabetes on basal insulin analogues: missed, mistimed and reduced doses. Curr Med Res Opin. 2012;28(12):1933-1946.

12. Jacqueminet S, Masseboeuf N, Rolland M, Grimaldi A, Sachon C. Limitations of the so-called "intensified" insulin therapy in type 1 diabetes mellitus. Diabetes Metab. 2005;31(4 Pt 2):4S45-4S50.

13. Bradley C, Todd C, Gorton T, Symonds E, Martin A, Plowright R. The development of an individualized questionnaire measure of perceived impact of diabetes on quality of life: the ADDQoL. Qual Life Res. 1999;8(1-2):79-91.

14. Seaquist ER, Anderson J, Childs B, et al. Hypoglycemia and diabetes: a report of a workgroup of the American Diabetes Association and the Endocrine Society. Diabetes Care. 2013;36(5):1384-1395.

15. Institute of Health Information and Statistics of the Czech Republic [webpage on the Internet]. Péče o nemocné cukrovkou. Available from: http://www.uzis.cz/katalog/zdravotnicka-statistika/pece-nemocnecukrovkou. Accessed January 1, 2016.

16. Karter AJ, Ackerson LM, Darbinian JA, et al. Self-monitoring of blood glucose levels and glycemic control: the Northern California Kaiser Permanente Diabetes registry. Am J Med. 2001;111(1):1-9.

17. Miller KM, Beck RW, Bergenstal RM, et al; T1D Exchange Clinic Network. Evidence of a strong association between frequency of self-monitoring of blood glucose and hemoglobin A1C levels in T1D exchange clinic registry participants. Diabetes Care. 2013;36(7): 2009-2014.

18. Murata GH, Shah JH, Hoffman RM, et al; Diabetes Outcomes in Veterans Study (DOVES). Intensified blood glucose monitoring improves glycaemic control in stable, insulin-treated veterans with type 2 diabetes: the Diabetes Outcomes in Veterans Study (DOVES). Diabetes Care. 2003;26:1759-1763.
19. Rogvi S, Tapager I, Almdal TP, Schiøtz ML, Willaing I. Patient factors and glycaemic control - associations and explanatory power. Diabet Med. 2012;29(10):e382-e389.

20. Davidson MB. Insulin analogs - is there a compelling case to use them? No! Diabetes Care. 2014;37(6):1771-1774.

21. Grunberger G. Insulin analogs - are they worth it? Yes! Diabetes Care. 2014;37(6):1767-1770.

22. Singh AK, Gangopadhyay KK. Modern basal insulin analogs: an incomplete story. Indian J Endocrinol Metab. 2014;18(6):784-793.

23. Gordon J, Pockett RD, Tetlow AP, McEwan P, Home PD. A comparison of intermediate and long-acting insulins in people with type 2 diabetes starting insulin: an observational database study. Int J Clin Pract. 2010;64(12):1609-1618.

24. Meneghini L, Kesavadev J, Demissie M, Nazeri A, Hollander P. Oncedaily initiation of basal insulin as add-on to metformin: a 26-week, randomized, treat-to-target trial comparing insulin detemir with insulin glargine in patients with type 2 diabetes. Diabetes Obes Metab. 2013;15(8):729-736.

25. UK Hypoglycaemia Study Group. Risk of hypoglycaemia in types 1 and 2 diabetes: effects of treatment modalities and their duration. Diabetologia. 2007;50(6):1140-1147.

26. Donnelly LA, Morris AD, Frier BM, et al; DARTS/MEMO Collaboration. Frequency and predictors of hypoglycaemia in type 1 and insulin-treated type 2 diabetes: a population-based study. Diabet Med. 2005;22(6):749-755.

27. Kuznetsov L, Griffin SJ, Davies MJ, et al. Diabetes-specific quality of life but not health status is independently associated with glycaemic control among patients with type 2 diabetes: a cross-sectional analysis of the ADDITION-Europe trial cohort. Diabetes Res Clin Pract. 2014;104(2):281-287.
Open Access Journal of Clinical Trials

\section{Publish your work in this journal}

The Open Access Journal of Clinical Trials is an international, peerreviewed, open access journal publishing original research, reports, editorials, reviews and commentaries on all aspects of clinical trial design, management, legal, ethical and regulatory issues, case record form design, data collection, quality assurance and data auditing

\section{Dovepress}

methodologies. The manuscript management system is completely online and includes a very quick and fair peer-review system, which is all easy to use. Visit http://www.dovepress.com/testimonials.php to read real quotes from published authors. 